\title{
Fibroblast Growth Factor 23-Related Osteomalacia Caused by the Prolonged Administration of Saccharated Ferric Oxide
}

\author{
Sunao Yamamoto ${ }^{1}$, Yosuke Okada ${ }^{1}$, Hiroko Mori ${ }^{1}$, Seiji Fukumoto ${ }^{2}$ and Yoshiya Tanaka ${ }^{1}$
}

\begin{abstract}
A 44-year-old woman with iron deficiency anemia was on a continuous course of intravenous saccharated ferric oxide (SFO). She came to our hospital because of right hip joint pain. She was found to have hypophosphatemia caused by impaired phosphorus resorption and her fibroblast growth factor 23 (FGF-23) levels were elevated. Therefore, she was diagnosed with FGF-23-related osteomalacia due to SFO administration. Discontinuation of the SFO treatment rapidly improved the impaired phosphorus resorption and also normalized the blood levels of phosphorus and FGF-23. During the treatment with SFO, it is important to regularly measure the blood levels of phosphorus in order to prevent the occurrence of osteomalacia.
\end{abstract}

Key words: osteomalacia, saccharated ferric oxide, fibroblast growth factor 23

(Intern Med 51: 2375-2378, 2012)

(DOI: 10.2169/internalmedicine.51.7450)

\section{Introduction}

Although saccharated ferric oxide (SFO) is frequently used to treat iron deficiency anemia in Japan, reports have indicated that long-term treatment can lead to osteomalacia $(1,2)$. Fibroblast growth factor 23 (FGF-23) has recently been identified as a new factor involved in phosphorus regulation, and has been reported to be involved in various phosphorus-related metabolic disorders (3). We herein report a case of osteomalacia caused by long-term administration of SFO, which led to elevated blood FGF-23 levels, in which discontinuation of SFO ultimately both normalized the FGF-23 levels and mitigated the osteomalacia.

\section{Case Report}

The patient was a 44-year-old woman who had visited our hospital for a consultation, complaining of pain between the ribs and in the right hip joint. She had grown and developed normally as a child, and neither drank nor smoked. She had been sufficiently exposed to the sun. In her 20 s, she was diagnosed with iron deficiency anemia due to uterine fibroids. She could not take oral iron supplements because of the gastrointestinal side effects; therefore, her treatment has consisted of continuous intravenous SFO administration. When the anemia worsened in 2007, the SFO dose was increased to $120 \mathrm{mg} /$ week. Around November 2007, she developed pain between the ribs and in the right hip joint with no particular provocation, and by June 2008, she had difficulty walking because of the right hip joint pain. She had visited another hospital to undergo orthopedic surgery at that time. However, when blood testing revealed hypophosphatemia, she was admitted to our hospital to undergo a detailed examination.

The physical findings at the time of admission were as follows: height, $159.8 \mathrm{~cm}$ (no loss of height); weight, 54.8 $\mathrm{kg}$; body temperature, $36.1^{\circ} \mathrm{C}$; and blood pressure, $109 / 69$ $\mathrm{mmHg}$. Her overall appearance did not indicate any bone deformity. Her palpebral conjunctivae appeared anemic, and there was tenderness around the right hip joint. Although she had received intravenous SFO until three weeks prior to the presentation, her blood findings showed iron-deficiency anemia (hemoglobin, $9.9 \mathrm{~g} / \mathrm{dL}$; mean corpuscular volume, $92.8 \mathrm{fL}$; serum iron, $27 \mu \mathrm{g} / \mathrm{dL}$; total iron binding capacity, $301 \mu \mathrm{g} / \mathrm{dL}$; transferrin saturation $9.0 \%$; ferritin, $58 \mathrm{ng} / \mathrm{mL}$ ).

The results of the biochemical analyses were as follows: calcium, $8.2 \mathrm{mg} / \mathrm{dL}$; phosphorus, $1.1 \mathrm{mg} / \mathrm{dL}$; intact parathy-

${ }^{1}$ The First Department of Internal Medicine, School of Medicine, University of Occupational and Environmental Health, Japan and ${ }^{2}$ Division of Nephrology and Endocrinology, Department of Medicine, University of Tokyo, Japan Received for publication January 31, 2012; Accepted for publication June 17, 2012 Correspondence to Dr. Yoshiya Tanaka, tanaka@med.uoeh-u.ac.jp 
(a)

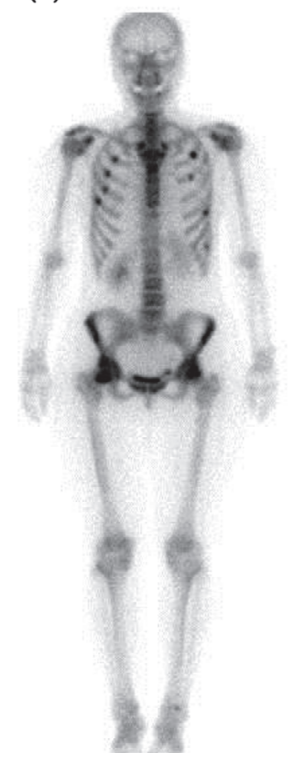

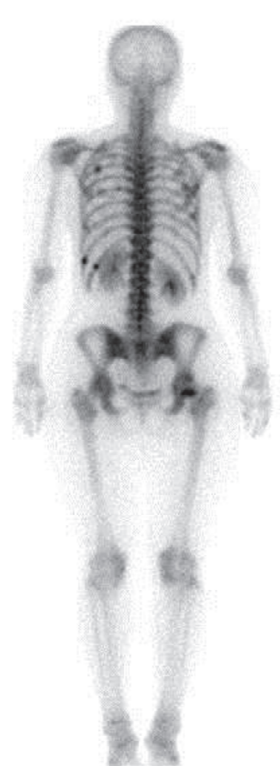

(b)

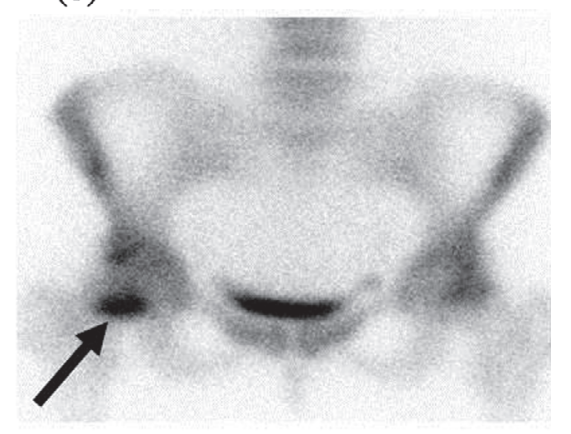

Figure 1. Abnormal accumulation in the ribs and right femoral neck (arrow).

roid hormone, $106 \mathrm{pg} / \mathrm{mL}$, which indicated secondary hyperparathyroidism. It is noteworthy that, despite the presence of hypophosphatemia, the maximal tubular phosphate reabsorption corrected for the glomerular filtration rate (TmP/GFR) was low at $0.95 \mathrm{mg} / \mathrm{dL}$, the 1,25-dihydroxyvitamin $[1,25$ (OH) 2D] level was relatively low at $20 \mathrm{pg} / \mathrm{mL}$, and FGF-23 was elevated at $30.2 \mathrm{pg} / \mathrm{mL}$. She was also vitamin $\mathrm{D}$ deficient, with levels of 25-hydroxyvitamin D [25(OH)D] at 14 $\mathrm{ng} / \mathrm{mL}$. In terms of bone metabolism markers, the levels of alkaline phosphatase (206 IU/L), bone-specific alkaline phosphatase $(24.7 \mathrm{ng} / \mathrm{mL})$, and urinary type I collagen cross-linked N-telopeptide (27.1 nM for bone collagen equivalents/mM creatinine) were all within the normal physiological ranges.

During the imaging studies, bone X-ray radiography (vertebra, thigh bone) revealed no evidence of bone thinning or fractures, and dual-energy X-ray absorptiometry of the lumbar vertebrae showed that the bone density was preserved, with a bone mineral density of $1.067 \mathrm{~g} / \mathrm{cm}^{2}$ and a T-score of 0.5 . On the other hand, bone scintigraphy showed abnormal accumulation in the ribs and right femoral neck consistent with the sites of pain (Fig. 1), and magnetic resonance imaging (MRI) also showed a fractured right femoral neck (Fig. 2).

The above results suggested hypophosphatemic osteomalacia caused by SFO, and the first step of treatment was to discontinue the SFO. Two weeks after discontinuation of SFO, the TmP/GFR had normalized to $4.20 \mathrm{mg} / \mathrm{dL}$ and the blood phosphorus levels had improved to $1.9 \mathrm{mg} / \mathrm{dL}$. Moreover, two months after SFO had been discontinued, the phosphorus level was found to have normalized to $3.5 \mathrm{mg} /$ $\mathrm{dL}$, while the FGF-23 level had also normalized to $8.6 \mathrm{pg} /$ $\mathrm{mL}$. These findings suggest that, at the time of admission, transitional secretion of FGF-23 was caused by SFO, and this had been involved in the hypophosphatemia. Nine months after SFO had been discontinued, the femoral neck fractures had been improved, as determined by MRI (Fig. 2). At that time, the patient's pain had almost disappeared, and she was able to continue walking and engaging in her hobby, golf, without difficulty. In addition, her 1,25 $(\mathrm{OH}) 2 \mathrm{D}$ level was normalized to $53.8 \mathrm{pg} / \mathrm{mL}$, suggesting that FGF-23 leads to a decrease in the serum $1,25(\mathrm{OH}) 2 \mathrm{D}$ levels. On the other hand, the low 25(OH)D level persisted unaltered, suggesting that her vitamin D deficient state was independent of the effects of SFO. She also underwent a radical hysterectomy to treat the uterine fibroids causing her iron-deficiency anemia.

\section{Discussion}

Osteomalacia is the pathological state of decreased bone strength occurring when vitamin D deficiency or chronic hypophosphatemia impairs bone calcification (4). In cases with adult-onset, the disease causes pain in the musculoskeletal system and decreases muscle strength, and bone fractures and deformities can develop as the disease progresses. Although no bone biopsy was taken in the present case, a clinical diagnosis of osteomalacia was reached on the basis of the blood levels of calcium, phosphorus, the bone scintigraphy findings, and the MRI findings of the hip joint.

The known factors causing osteomalacia include insufficient dietary vitamin D intake, vitamin D dependence, and chronic hypophosphatemia caused by renal tubular damage (4). Some reports have implicated the long-term administration of SFO and iron polymaltose as being iatrogenic causes of osteomalacia via the induction of chronic hypophosphatemia $(1,2,5)$. SFO has been suggested to cause hypophosphatemia via decreased phosphorus resorption by the renal tubules, thus resulting in it being filtered out from the glomeruli and deposited in the tubular epithelium, pro- 
(a)

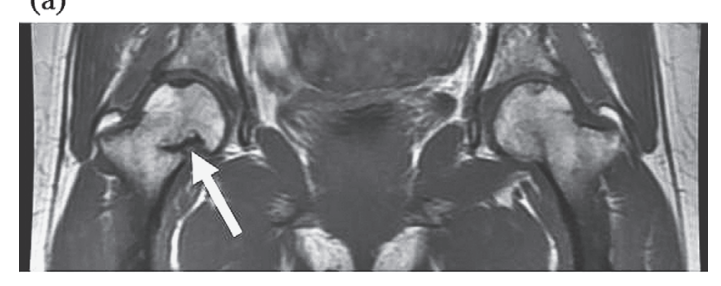

(b)

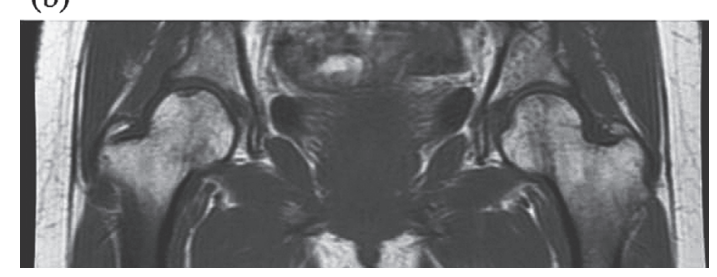

Figure 2. (a) Before treatment. Fractured right femoral neck (arrow). (b) Nine months after SFO was discontinued. Improvement in fracture site.

voking tubular damage (6). However, histological testing in rat and human kidneys has not found iron deposition or other abnormalities in the tubules, leading to the hypothesis that there is a mechanism other than decreased phosphorus resorption that underlies this condition $(7,8)$.

FGF-23 has been identified to be a new peptide that acts as a phosphorus regulation factor (9). It is composed of 227 amino acids, and is secreted from osteocytes. It inhibits phosphorus resorption by decreasing the expression of type IIa and type IIc Na-P cotransporters in the renal proximal tubules (10). FGF-23 also decreases the expression of 25 $(\mathrm{OH}) \mathrm{D}-1 \alpha$-hydroxylase, which is a vitamin D-metabolizing enzyme in the renal tubules, while also promoting the expression of $25(\mathrm{OH}) \mathrm{D}-24$-hydroxylase. This leads to a decrease in the $1,25(\mathrm{OH}) 2 \mathrm{D}$ levels in the blood, and subsequently decreases the phosphorus absorption by the bowel (10).

Accordingly, an excessive expression of FGF-23 over the long term can lead to hypophosphatemic osteomalacia (FGF-23-related osteomalacia). The types of FGF-23-related osteomalacia include tumor-induced osteomalacia, X-linked hypophosphatemic osteomalacia, autosomal dominant and recessive hypophosphatemic osteomalacia, McCune-Albright syndrome, and hypophosphatemic osteomalacia caused by fibrous dysplasia (3). Recent reports have shown that intravenous administration of SFO elevates the FGF-23 concentration in the blood and can lead to the onset of hypophosphatemic osteomalacia, and SFO-induced osteomalacia has been categorized as a FGF-23-related osteomalacia (11). Because hypophosphatemic osteomalacia is provoked by neither chondroitin sulfate iron colloid nor cideferron, which are also intravenous iron supplements, it seems unlikely that it is the iron itself that is active in promoting FGF-23 secretion, and much remains unclear about the mechanism underlying the condition. The present case also exhibited hypophosphatemia and an inappropriate amount of FGF-23 secretion following the intravenous administration of SFO. The fact that the phosphorus levels and FGF-23 secretion quickly normalized after the discontinuation of SFO suggest that this was a case of FGF-23-related osteomalacia caused by SFO administration. When hypophosphatemia is present with a value of less than $2.5 \mathrm{mg} / \mathrm{dL}$, if the level of FGF-23 is higher than $30 \mathrm{pg} / \mathrm{mL}$, the condition is diagnosed as FGF23-related hypophosphatemia (12).

The present case also included confirmation of a decreased 25(OH)D level in the blood, which persisted for nine months after SFO was discontinued. This suggests that the circumstances of the present case included a vitamin D deficiency that was independent of the dynamics of FGF-23. We explored the possibility of administering activated vitamin D, but given that the patient's symptoms had improved and it was undesirable to begin administration of a new drug, a dietary treatment was introduced instead, and her progress is currently being monitored.

We herein summarized our experience with a case in which long-term administration of SFO had led to FGF-23related osteomalacia. SFO is frequently used to treat iron deficiency anemia, but appropriate management must be undertaken to ensure bone health, and the treatment should be discontinued if necessary. If long-term administration is unavoidable, it is very important to utilize the minimum required dose and for the shortest duration necessary, and to regularly measure the blood phosphorus levels to avoid the onset of osteomalacia.

The authors state that they have no Conflict of Interest (COI).

\section{References}

1. Okada M, Imamura K, Iida M, Fuchigami T, Omae T. Hypophosphatemia induced by intravenous administration of Saccharated iron oxide. Klin Wochenschr 61: 99-102, 1983.

2. Sato K, Shiraki M. Saccharated ferric oxide-induced osteomalacia in Japan: iron-induced osteopathy due to nephropathy. Endocr J 45: 431-439, 1998.

3. Hori M, Shimizu Y, Fukumoto S. Minireview: fibroblast growth factor 23 in phosphate homeostasis and bone metabolism. Endocrinology 152: 4-10, 2011.

4. Bringhurst FR, Demay MB, Krane SM, Kronenberg HM. Bone and mineral metabolism in health and disease. In: Harrison's Principles of Internal Medicine. 17th ed. Fauci AS, Braunwald E, Kasper DL, et al, Eds. McGraw-Hill, New York, 2008: 2365-2377.

5. Schouten BJ, Hunt PJ, Livesey JH, Frampton CM, Soule SG. FGF23 elevation and hypophosphatemia after intravenous iron polymaltose: a prospective study. J Clin Endocrinol Metab 94: 2332-2337, 2009.

6. Kirschbaum BB. Aggregation of renal brush border membranes by concanavalin A and heavy metals. Toxicol Appl Pharmacol 64: 10-19, 1982.

7. Sanai T, Oochi N, Okada M, Imamura K, Okuda S, Iida M. Effect of saccharated ferric oxide and iron dextran on the metabolism of phosphorus in rats. J Lab Clin Med 146: 25-29, 2005.

8. Imamura K. Effects of intravenous administration of iron preparations on the metabolism of phosphorus. Comparative study on 3 iron preparations. Fukuoka Igaku Zasshi 75: 316-326, 1984.

9. Yamashita T, Yoshioka M, Itoh N. Identification of a novel fibroblast growth factor, FGF-23, preferentially expressed in the ven- 
trolateral thalamic nucleus of the brain. Biochem Biophys Res Commun 277: 494-498, 2000.

10. Shimada T, Hasegawa H, Yamazaki Y, et al. FGF-23 is a potent regulator of vitamin $\mathrm{D}$ metabolism and phosphate homeostasis. J Bone Miner Res 19: 429-435, 2004.

11. Shimizu Y, Tada Y, Yamauchi M, et al. Hypophosphatemia induced by intravenous administration of saccharated ferric oxide: another form of FGF23-related hypophosphatemia. Bone 45: 814816, 2009.

12. Endo I, Fukumoto S, Ozono K, et al. Clinical usefulness of measurement of fibroblast growth factor 23 (FGF23) in hypophosphatemic patients: proposal of diagnostic criteria using FGF23 measurement. Bone 42: 1235-1239, 2008.

(C) 2012 The Japanese Society of Internal Medicine http://www.naika.or.jp/imonline/index.html 